\title{
Polímeros Luminescentes como Sensores de Radiação não Ionizante: Aplicação em Fototerapia Neonatal
}

\author{
Cláudia K. B. de Vasconcelos, Rodrigo F. Bianchi \\ Instituto de Ciências Exatas e Biológicas, UFOP
}

\begin{abstract}
Resumo: Neste trabalho foram observadas mudanças nas propriedades de emissão e de absorção de soluções de poli[2metoxi-5-(2'etil-hexiloxi)-p-fenilenovinileno] - MEH-PPV com a exposição à radiação na região espectral do visível na faixa de 425 a $500 \mathrm{~nm}$. Os resultados demonstram a possibilidade do uso de sistemas luminescentes como elemento ativo de detectores de radiação não ionizante, sobretudo na região empregada na profilaxia e no tratamento fototerápico da hiperbilirrubinemia neonatal.
\end{abstract}

Palavras-chave: Polímero, sensor, radiação, fototerapia, icterícia, neonatal. hiperbilirrubinemia.

\section{Luminescent Polymers as Sensor for Non-ionizing Radiation: Neonatal Phototherapy Application}

Abstract: In this work, we observed changes in the emission and absorption properties of poly[(2-methoxy-5-hexyloxy)-pphenylenevinylene] - MEH-PPV solutions in the visible range from 425 to $500 \mathrm{~nm}$. The main results demonstrated the possibility of using luminescent polymers as active material of non-ionizing radiation dosimeter, in such a way that MEH-PPV is promising for use in a management system to monitor radiation absorbed by newborns during phototherapy treatment of neonatal hyperbilirubinemia.

Keywords: Polymer, sensor, radiation, phototherapy, neonatal, hyperbilirubinemia, jaundice.

\section{Introdução}

A partir da descoberta das propriedades luminescentes do poli(p-fenilenovinileno) - PPV em $1990^{[1]}$, inúmeros grupos de pesquisa e indústrias do mundo começaram a explorar a possibilidade de fabricação de displays luminosos poliméricos de baixo custo, de baixo consumo de energia e de fácil fabricação ${ }^{[2]}$. Dos anos seguintes a esta descoberta, até os dias de hoje, diversos polímeros e protótipos de dispositivos luminosos foram obtidos, destacando-se os PLEDs (polymer light-emitting dio$d e s)^{[3]}$ e os displays policromáticos ${ }^{[4,5]}$. Entretanto, apesar do estágio atual de desenvolvimento tecnológico destes dispositivos ${ }^{[6]}$, os fenômenos responsáveis pela eficiência luminosa e pelo tempo de vida destes sistemas ainda são pouco compreendidos ${ }^{[7,8]}$. Desta forma, enquanto as promessas de novos dispositivos crescem a cada dia, o fraco desempenho destes sistemas tem inviabilizado muitas de suas aplicações comerciais ${ }^{[8]}$. Muitos autores têm estudado e se preocupado com este tema, propondo que a otimização da eficiência luminoso e do tempo de vida dos dispositivos só será possível quando os mecanismos ligados à sua degradação forem entendidos e minimizados ${ }^{[8,9]}$. Dentre estes mecanismos, destaca-se a fotoxidação da camada polimérica ${ }^{[9,10]}$. Devido às presenças de luz e oxigênio, inerentes aos processos de fabricação e/ou às condições de operação dos dispositivos, ligações vinílicas $(\mathrm{C}=\mathrm{C})$ das cadeias poliméricas são substituídas por ligações carbonilas
$(\mathrm{C}=\mathrm{O})^{[9,10]}$. Assim, o comprimento da conjugação da cadeia principal é reduzido, modificando as propriedades eletrônicas dos polímeros e influenciando diretamente o desempenho dos seus dispositivos luminosos ${ }^{[8,11]}$. Se do ponto de vista da tecnologia de displays o fenômeno da fotoxidação tem limitado a comercialização destes sistemas, este fenômeno surge aqui como uma importante característica para o desenvolvimento de novos dispositivos eletrônicos, onde a variação das propriedades ópticas e elétricas dos polímeros sob exposição à radiação torna-se um aspecto de grande interesse científico e tecnológico ${ }^{[12,13]}$. Em particular, o crescente número de estudos na área de radiação não ionizante encontra justificativa tanto nos malefícios causados à saúde das pessoas por exposição a este tipo de radiação, como a ocorrência do câncer de pele, bem como, por exemplo, em tratamentos da hiperbilirrubinemia neonatal, freqüentemente utilizados na prática clínica diária em Neonatologia. Por este motivo, existe uma demanda cada vez maior por métodos capazes de quantificar e monitorar a dose de radiação emitida por uma determinada fonte com a presteza, segurança e eficiência necessárias à saúde a ao bem estar. Ainda mais interessante é o fato dos espectros de absorção na região do visível de derivados do PPV, como o poli[2-metóxi-5-(2'etil-hexilóxi)- $p$ fenilenovinileno] - MEH-PPV, apresentarem grandes variações quando o polímero é exposto a baixas doses de radiações ionizantes ou não ${ }^{[13-16]}$. Tal comportamento abre, portanto, a possibilidade de confecção de dosímetros de 
grande potencial para uso na área médica, não apenas no controle de exposição à radiação de pacientes neonatais ${ }^{[17]}$, como também das taxas de exposições excessivas dessa radiação com propósitos de bronzeamento artificial ${ }^{[18]}$; ou ainda, no setor de segurança do trabalho, no controle das taxas de exposição dos trabalhadores civis e rurais ${ }^{[19]}$ à radiação ultravioleta.

Neste trabalho é apresentado o estudo do comportamento das propriedades ópticas de soluções de MEH-PPV submetidos a diferentes doses de radiação na região do visível tal como utilizadas no tratamento da hiperbilirrubinemia neonatal.

\section{Experimental}

Nesse trabalho foi utilizado o poli[2-metóxi,5(2'etilhexiloxi)-p-fenilenovinileno] - MEH-PPV dissolvido em clorofórmio $\left(\mathrm{CHCl}_{3}\right)$ com concentração de $0,025 \mathrm{mg} / \mathrm{ml}$, de acordo com os resultados prévios e os procedimentos adotados por J. F. Borin ${ }^{[13]}$ recentemente. O sistema, uma vez preparado, foi exposto a diferentes tempos de radiação na região do visível, de 425 a $500 \mathrm{~nm}$, obtida por meio do uso de LEDs azuis próprios para o tratamento de hiperbilirrubinemia ${ }^{[20]}$, mantendo-se a temperatura em torno de $27{ }^{\circ} \mathrm{C}$. Durante o procedimento de radiação, soluções com $2 \mathrm{ml}$ foram mantidas em ampolas de vidro, cujas pontas capilares foram lacradas por um maçarico (de maneira afótica) para evitar a evaporação do solvente e, conseqüentemente, evitar mudanças na concentração da solução durante as medidas. A Figura 1a mostra o sistema constituído pelas ampolas de vidro preenchidas com a solução do polímero luminescente e submetidas a diferentes tempos de exposição à radiação. Nesse caso, a coloração do material muda do vermelho para o transparente, passando pelo laranja e depois pelo amarelo, à medida que a amostra vai sendo exposta à luz de um LED azul com ângulo de visão, luminosidade e pico de emissão em torno de, respectivamente, $45^{\circ}, 4000 \mathrm{mcd}$ e $475 \mathrm{~nm}$. A Figura 1b, por sua vez mostra o espectro de emissão do LED utilizado no trabalho.

As ampolas contendo as soluções de MEH-PPV, por sua vez, foram caracterizadas por meio de espectroscopia de absorção do ultravioleta visível (UV-VIS) e de fotoemissão com o auxílio de um espectrofotômetro OCEAN OPTICS USB2000. Ambas foram realizadas simultaneamente por meio da exposição do polímero a intensidade luminosa de um LED branco. Nesse caso, devido às características eletrônicas do MEH-PPV ${ }^{[21]}$, a resposta espectral proveniente da solução, que é apresentada na Figura 2, é composta não apenas da luz absorvida pela solução na faixa de 400 a $550 \mathrm{~nm}$, como também da contribuição da luz fotoemitida pelo material na região de 500 a $650 \mathrm{~nm}^{[20]}$.

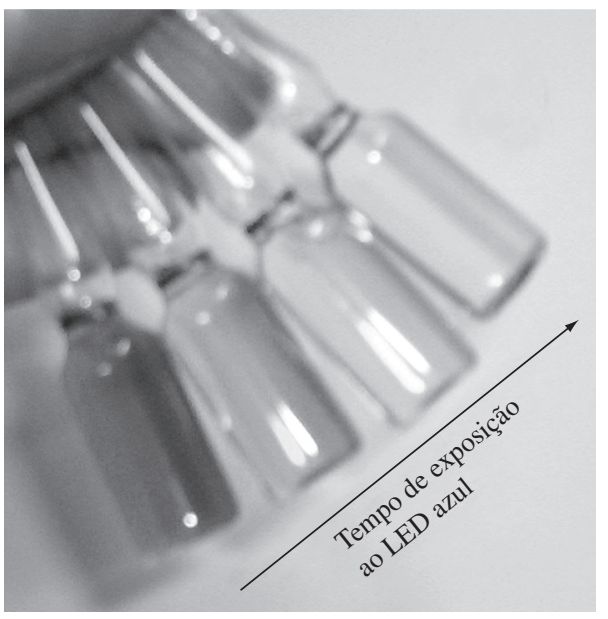

(a)

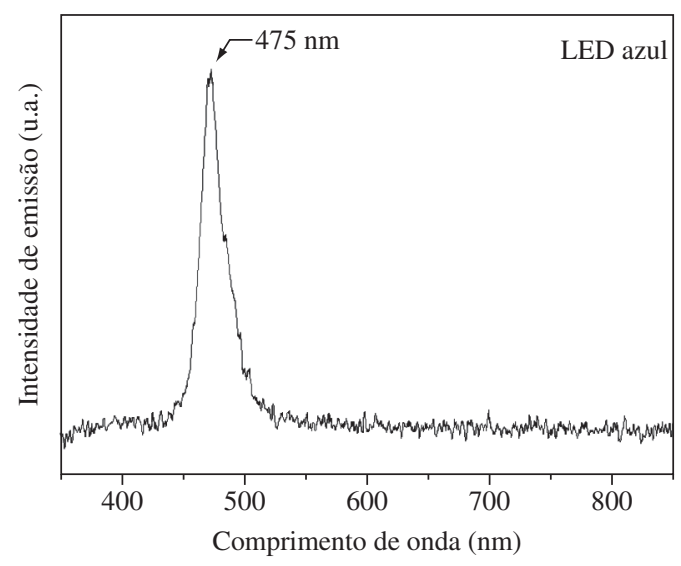

(b)

Figura 1. a) sistema constituído de ampola e solução de MEH-PPV em $\mathrm{CHCl}_{3}$ utilizada como sensor de radiação não ionizante. A coloração da solução polimérica muda do vermelho para o transparente, passando pelo laranja e pelo amarelo, à medida que a solução vai sendo exposta à luz do LED azul; e b) espectro de emissão do LED utilizado no trabalho.

\section{Resultados e Discussão}

A Figura 2 mostra as respostas espectrais obtidas com a solução de MEH-PPV em $\mathrm{CHCl}_{3}$ exposta ao LED azul.

Dos resultados mostrados na Figura 2 observa-se que à medida que a solução é exposta ao LED, a intensidade da luz proveniente da solução diminui em toda faixa de comprimento de onda investigado, e o pico de máxima intensidade, que se encontra inicialmente em torno de $500 \mathrm{~nm}$, desloca-se para um valor próximo de $450 \mathrm{~nm}$ após $570 \mathrm{~min}$, com intensidade luminosa reduzida a $65 \%$ do seu valor inicial. Ademais, um pico de mínimo de intensidade, em torno de $550 \mathrm{~nm}$ e, portanto, dentro da faixa de emissão da amostra, passa a se tornar mais pronunciado com a exposição da solução à luz. Tal resultado deixa claro que a exposição do polímero ao LED azul age no sentido de diminuir tanto a intensidade de absorção quanto a de emissão da amostra. Não obstante tal mudança de intensidade dos espectros de absorção com o tempo de exposição, há de se levar em consideração que o comprimento de onda 


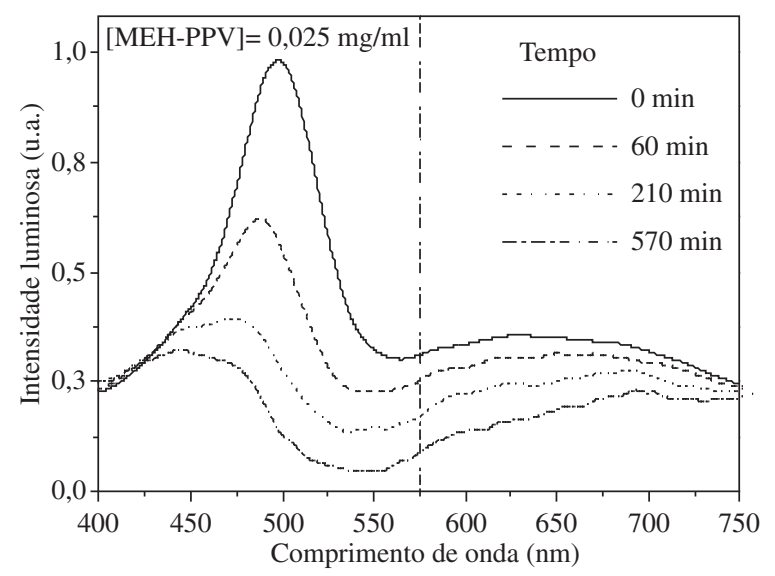

Figura 2. Resposta espectral (composição dos espectros de absorção e de fotoemissão) da solução de $\mathrm{MEH}-\mathrm{PPV}$ em $\mathrm{CHCl}_{3}$ exposta a diferentes tempos de radiação não ionizante proveniente de um LED azul usado no tratamento da hiperbilirrubinemia.

máximo $\left(\lambda_{\text {máx }}\right)$ desses espectros também varia com o tempo, deslocando-se para o azul, como mostra a Figura 3. Resultados similares foram obtidos recentemente por Boriin ${ }^{[13]}$, Bianchi ${ }^{[9]}$, Olivati ${ }^{[22]}$ e colaboradores no estudo do comportamento das propriedades ópticas de soluções e/ou filmes de MEH-PPV expostos a diferentes tempos de radiações ionizante ou não. Os resultados obtidos por esses autores mostram que as mudanças nos espectros de absorção e de fotoemissão do polímero, sobretudo quando exposto a radiação na região do visível, está relacionada ao processo de fotoexcitação do material devido à quebra das ligações vinílicas, e conseqüente incorporação de ligações carbonílicas $(\mathrm{C}=\mathrm{O})$, na cadeia polimérica principal, reduzindo tanto a intensidade de absorção dos espectros, como também os seus $\lambda_{\text {máx }}$ 's. Finalmente, o comportamento de $\lambda_{\text {máx }}$ $v s$. tempo é importante para a proposta de trabalhos futuros focados na confecção de dispositivos eletrônicos de controle e de monitoramento de sensores de radiação de MEH-PPV, uma vez que desse resultado é permitido analisar não somente a faixa de operação do sensor (de 0 a 570 min), como também a sua resposta intensidade-tempo.

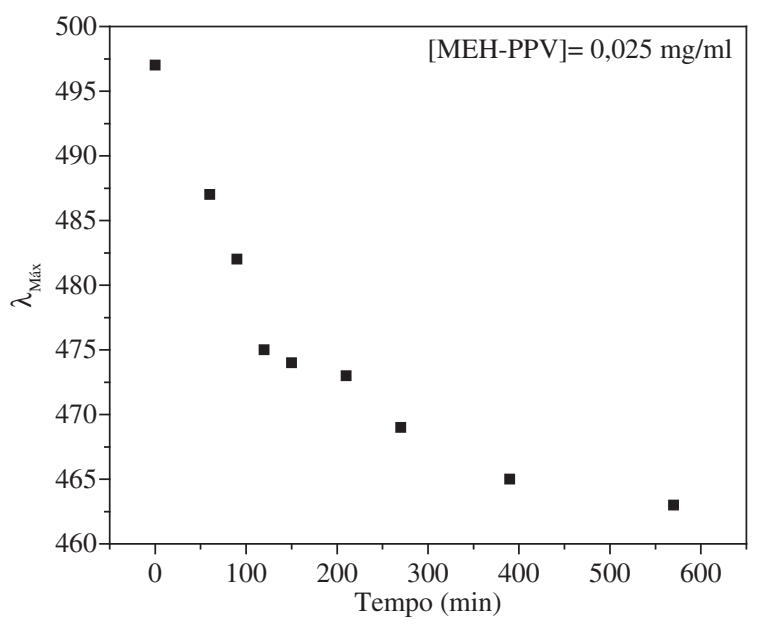

Figura 3. Comprimento de onda máximo $\left(\lambda_{\max }\right) v s$. tempo de radiação absorvida pelo MEH-PPV obtidos dos gráficos apresentados na Figura 3.

\section{Conclusões}

O tratamento da hiperbilirrubinemia neonatal com radiação proveniente de um LED azul é um tema atual e bastante atraente, não apenas pela facilidade e pelo baixo custo do tratamento médico a que se refere, mas, principalmente, pela sua eficiência e simplicidade. O mesmo acontece com o uso de polímeros luminescentes como elementos ativos de sensores de radiação. Tais materiais, amplamente utilizados em displays luminosos, têm o efeito da fotoxidação como um dos seus principais obstáculos para inserção comercial. Nesse sentido, esse trabalho utiliza o efeito deletério da degradação do MEH-PPV usado em displays como principal agente no sentido de se obter um material eficiente para o monitoramento e controle da dose/tempo de radiação absorvida por doentes expostos a procedimentos de fototerapia neonatais. Para tanto, foram realizadas medidas de absorção e de fotoemissão de soluções de $\mathrm{MEH}-\mathrm{PPV}$ em $\mathrm{CHCl}_{3}$, previamente expostas a diferentes tempos de radiação com um LED azul. Os resultados obtidos mostraram um deslocamento dos máximos de absorção e emissão para o azul que é característico de processos de fotoxidação da cadeia polimérica principal do MEH-PPV.

\section{Agradecimentos}

Os autores agradecem ao CNPq e à FAPEMIG pelo suporte financeiro.

\section{Referências Bibliográficas}

1. Burroughes, J. H.; Bradley, D. D. C.; Brown, A. R.; Marks, R. N.; Mackay, K.; Friend, R. H.; Burns, P. L. \& Holmes, A. B - Nature 347, 539 (1990).

2. Nalwa, H. S. - "Organic Conductive Molecules and Polymers", John Wiley \& Sons, $1^{\text {st }}$ Ed. (1997).

3. Friend, R. H.; Gymer, R. W.; Holmes, A. B.; Burroughes, J. H.; Marks, R. N.; Taliani, C.; Bradley, D. D. C; Dos Santos, D. A.; Bredas, J. L.; Logdlund, M. \& Salaneck, W. R. - Nature 397, 6715, 121 (1999).

4. Pope, M. \& Swenberg C.E. - in: "Electronic process in organic crystlas and polymers", Clarenfon Press, $2^{\text {nd }}$ Ed., Oxford Press (1999)

5. Friend, R.; Burroughes, J. \& Shimoda, T. - Phys. Word 12, 6, 35 (1999).

6. Howard, W. E. - Sci. American Brasil 2, 22, 86 (2004).

7. Brütting, W.; Berleb, S. \& Mückl, A. - Org. Elec. 2, 1 (2001).

8. Fordonar, R. - Newsweek, 23 February (2004).

9. Bianchi, R. F.; Balogh, D. T.; Tinani, M.; Faria, R. M. \& Irene, E.A. - J. Pol. Sci. Part B: Pol. Phys. 42, 6, 1033 (2004). 
10. Janssen, F. J. J.; Van IJzendoon, L. J.; Schoo, H. F. M.; Sturm, J. M.; Andersson, G. G.; Van der Gon, A. W. D.; Brongersma, H. H. \& de Voigt, M. J. A. - Synt. Met. 131, 1-3, 167 (2002).

11. Low H. Y. - Thin Sol. Films 413, 1-2, 160 (2002).

12. Silva, E. A. B. - "Estudo das características de polímeros submetidos à radiação e sua aplicação como dosímetro," Dissertação de Mestrado, Faculdade de Filosofia, Ciências e Letras de Ribeirão Preto, Universidade de São Paulo, Brasil (2004).

13. Borin, J. F.; Silva, E. A. B; Nicolucci, P.; Netto, T. G.; Graeff, C. F. O. \& Bianchi, R. F. - Appl. Phys. Let. 86, 131902 (2005).

14. Bianchi, R. F. - "Estudo das propriedades eletrônicas e ópticas de filmes e dispositivos poliméricos", Tese de Doutorado, Instituto de Física de São Carlos, Universidade de São Paulo, Brasil, (2002).

15. Hioki, E. T. “Confecção e caracterização de polímeros luminescentes submetidos à radiação e sua aplicação em dosímetros", Iniciação Científica, Proc. Fapesp No 0/07719-1, Out/2004-Set/2005.

16. Graeff, C. F. O.; Borin, J. F.; Brito, E. S. \& Bianchi, R. F. - "Dosímetro de Radiação Ionizante de Baixa Dose Construído com Polímero Conjugado". Patente de In- venção, PI 0600986-7, Instituto Nacional da Propriedade Industrial (2006).

17. Berkow, R. - "Saúde para a Família", Manual Merck, Seção 23, Cap. 252 (2005).

18. Chaves, A.; Shellard, R. C. - "O desenvolvimento da Física e sua inserção na vida social e econômica do país", Sociedade Brasileira de Física, p. 166-167 (2005).

19. Instituto Nacional de Câncer (Brasil). Prevenção e controle do câncer: normas e recomendações do INCA. Rev Bras Cancerol, 48, p.317-32 (2002).

20. Morais, B. M. - "Guia de Pediatria: Tratamento", UNIFESP - Escola Paulista de Medicina, Cap. 34, p.57-64 (2005).

21. Vasconcelos, C. K. B. \& Bianchi, R. F. - "Dosímetro de radiação não ionizante construído com polímeros conjugado". Patente Requerida, 0000220700175455/INPI (2007).

22. Olivati, C.A.; Ferreira, M.; Bianchi, R. F.; Faria, R. M.; Osvaldo, O. N. \& Balogh, D.T. - Pol. Deg. Stab. 91,10 2342 (2006).

Enviado: 14/02/07

Reenviado: $27 / 04 / 07$

Aceito: $31 / 05 / 07$ 